\title{
Probing the energy bands of a Bose-Einstein condensate in an optical lattice
}

\author{
M. L. Chiofalo, ${ }^{1}$ S. Succi, ${ }^{2}$ and M. P. Tosi ${ }^{1}$ \\ ${ }^{1}$ INFM and Classe di Scienze, Scuola Normale Superiore, I-56126 Pisa, Italy \\ ${ }^{2}$ Istituto Applicazioni Calcolo "M. Picone" and INFM, I-00161 Roma, Italy
}

(Received 23 January 2001; published 17 May 2001)

\begin{abstract}
We simulate several methods which could be realized in the laboratory to probe the band excitation energies and the momentum distribution of a Bose-Einstein condensate inside an optical lattice. The values of the excitation energies obtained by the different methods agree within the accuracy of the simulation. The meaning of the results in terms of density and phase deformations is tested by studying the relaxation of a phasemodulated condensate toward the ground state.
\end{abstract}

DOI: 10.1103/PhysRevA.63.063613

PACS number(s): 03.75.Fi, 32.80.Pj, 67.40.Db

\section{INTRODUCTION}

A periodic potential can be imposed on a cold atomic gas from a shift of the atomic ground state under illumination by a detuned laser standing wave [1]. Experiments on ultracold atoms inside such an optical lattice have revealed dynamical behaviors which are well known from band theory for electrons in solids, i.e., Wannier-Stark ladders [2], Bloch oscillations [3], and Landau-Zener tunneling [4]. Especially exciting perspectives are offered by the confinement of an atomic Bose-Einstein condensate (BEC) inside an optical lattice, in regard to the generation of coherent matter-wave pulses [5], laser cooling [6], quantum computing [7] and more generally BEC quantum-state engineering.

Theoretical and numerical studies of a BEC in a periodic potential have been based on solutions of the static Bogolubov-de Gennes equations [8-11] and the timedependent Gross-Pitaevskii equation (GPE) [12-16]. These studies showed that the phase coherence of the condensate distinguishes it from an assembly of ultracold atoms under essential aspects concerning both the energy-band structure and the transport behavior.

As to the band structure, the main, and most striking consequence, of BEC phase coherence shows up in the nature of the excitations in the various energy bands. Excitations in the lowest band arise from pure phase modulations of the condensate with a given $q$ vector in the Brillouin zone, whereas the higher bands are associated with density profiles having the symmetry of the higher isolated-well states (see, especially, Ref. [11], where these properties are derived using the Wannier representation for the wave functions). Second, a phononlike linear dispersion relation may be expected in the lowest band at the Brillouin-zone center [8,11]. This property can be traced back to the presence of the mean-field interactions combined with the structure of the Bogolubov-de Gennes equations under time-reversal invariance. In solid-state physics, a similar behavior is found for electrons in the band structure of graphite, from the mixing of atomic orbitals, to give wave functions which are even or odd under time reversal. A condensate in a periodic potential may show such a linear dispersion, depending on the relative strength of the mean-field vs lattice interactions, or equivalently on the ratio of the healing length to the lattice spacing.

As to the transport behavior, a BEC subject to a periodic optical potential and to a constant external force performs
Bloch oscillations as if it were a quasi particle inside the lowest-energy band. At variance from ultracold atomic gases, such a control of the sharpness of the momentum distribution is essential in the aforementioned physical applications and, as an example, is consistent with the observed emission of coherent matter pulses from a vertical array of optical traps under the force of gravity [5].

The present work examines some pump-probe experiments which could be performed to explore the nature of condensate excitations inside an optical lattice under currently attainable experimental conditions, and to expose its quasiparticle behavior from its momentum distribution. To this end, after introducing the numerical methods used to solve the GPE in Sec. II, we simulate two different pumpprobe experiments based on techniques which have already been realized in the laboratory to measure Bloch oscillations of ultracold Bose atoms [3] and to study the shape deformation modes of a BEC in harmonic traps $[17,18]$. In the first method, described in Sec. III A, a velocity is imparted to the whole condensate at time $t=0$ and the velocity spectrum is thereby probed, while in the second (see Sec. III B) a resonant parametric driving of the optical lattice is carried out for a variable length of time and the density spectrum is determined from an analysis of the condensate dynamics. In each method we discuss the physical content of the pumping mechanism and of the observable quantities, in order to infer the nature of the energy bands as noted above. We further test the meaning of these concepts in Sec. III C, where we use the propagation in imaginary time of an initially phasemodulated condensate to probe the excitations through their interband and intraband relaxations to equilibrium. The results of the different methods are then compared in Sec. III D, and displayed as energy bands.

In Sec. IV we illustrate the measurement of the velocity spectrum discussed in Sec. III A and the BEC coherent dynamical behavior under the influence of an external force, by simulating an experiment to measure the sharp momentum distribution of the condensate. Finally, we give our concluding remarks in Sec. V.

\section{SIMULATION METHOD}

We consider a dilute BEC in the external potential $U_{l}^{0}[1$ $\left.-\exp \left(-r^{2} / r_{l b}^{2}\right) \cos ^{2}(2 \pi z / \lambda)\right]$, where $U_{l}^{0}$ is the well depth, $\lambda$ and $r_{l b}$ are the laser-beam wavelength and waist, and $d$ 
$=\lambda / 2$ is the lattice period. Its dynamics is accurately described by a one-dimensional (1D) model, if the mean-field interactions are renormalized to reproduce the correct 3D value of the chemical potential [14]. We thus adopt the 1D GPE for the BEC wave function $\Psi(z, t)$ :

$$
i \hbar \frac{\partial \Psi(z, t)}{\partial t}=\left[-\frac{\hbar^{2} \nabla_{z}^{2}}{2 M}+U(z, t)+U_{p}(z, t)\right] \Psi(z, t) .
$$

In Eq. (1), $U(z, t)$ includes the 1D lattice potential $U_{l}(z)$ and the mean-field interactions, i.e., $U(z, t)=U_{l}^{0} \sin ^{2}(\pi z / d)$ $+4 \pi \hbar^{2} \gamma a \rho|\Psi(z, t)|^{2} / M$, with $a$ the scattering length, $\gamma$ the renormalization factor, $\rho$ the number of particles per lattice well, and $M$ the atom mass. We study three different forms for the pump potential $U_{p}(z, t)$, as will be specified below. We adopt the system parameters from the experiment on ${ }^{87} \mathrm{Rb}[5]\left(a=110\right.$ Bohr radii, $\lambda=850 \mathrm{~nm}$ and $U_{l}^{0}=1.4 E_{R}$, with $E_{R}=h^{2} / 8 M d^{2}$ ), and take $\rho=2500$ atoms per well.

On the technical side, we handle the heavy simulations needed to obtain significant results on fine spectral structures by a well-tested explicit-time-marching algorithm [15,19]. We first determine the ground state by numerically propagating Eq. (1) in imaginary time, and then insert it as the initial condition for evolution in real time. By the same algorithm we preliminarily calculate the band energies $E_{n}(q)$ from the relaxation of a statically deformed BEC (see below), $n$ being the band index and $q$ the reduced wave vector in the Brillouin zone. The size of the simulation box is 700 wells, as needed for a three-digit accuracy in the ground-state energy $\left(\mu=0.695 E_{R}\right)$. We guard against unwanted localized excitations at the boundaries by letting the density profile vanish over a length scale which is much larger than the healing length $\xi=(8 \pi a \rho)^{-1 / 2} \simeq 1.4 d$. Typically, a grid contains 5 $\times 10^{4}$ points, and the simulation is carried out up to final times ranging from 13 to $32 \mathrm{~ms}$. In dynamical simulations the data are stored every $20 \mu \mathrm{s}$, and used to obtain the real and imaginary parts of the Fourier transform of the wave function $[\Psi(z, \omega)$, say $]$.

\section{SIMULATION RESULTS}

\section{A. Probing the velocity spectrum}

In the first simulational method, which we familiarly refer to as kicking, we impart a velocity $v=\hbar q / M$ to the BEC at time $t=0$, by imposing a phase $q z$ on the ground-state wave function. The dynamics of the BEC is then monitored with $U_{p}=0$. We first analyze the dynamics of the wave function $\Psi(z, t)$, and then comment on its observability.

In Fig. 1 we show the spectra $\operatorname{Re} \Psi(z, \omega)$ obtained after Fourier transform of $\Psi(z, t)$ at any position $z$ in the bulk of the BEC, as functions of $(\hbar \omega-\mu) / E_{R}$ for four values of $q$. At each $q$ the strong peak on the left refers to excitations in the lowest band $(n=0$, say) and the other two peaks belong to the bands $n=1$ and 2 . The eigenfrequencies revealed by Fig. 1 are the same as those appearing in $\operatorname{Im} \Psi(z, \omega)$. The density spectrum $n_{c}(z, \omega)=\int d t \exp (i \omega t)|\Psi(z, t)|^{2}$, which is not displayed here, instead shows a strong peak centered at energy $\mu$ for all values of $q$ and two further peaks corre-

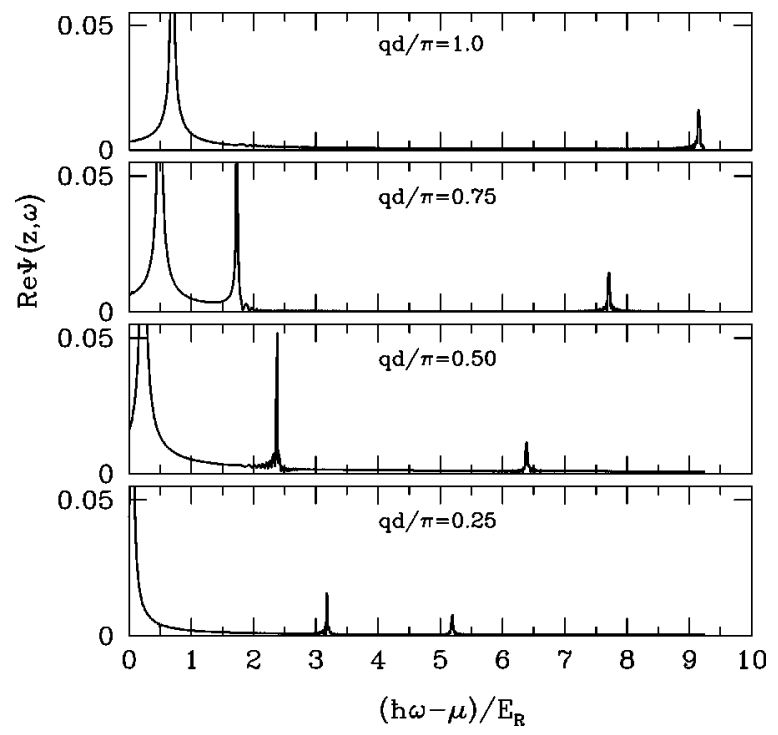

FIG. 1. Kicking method: bulk spectrum $\operatorname{Re} \Psi(z, \omega)$ as a function of $(\hbar \omega-\mu) / E_{R}$ for four values of $q$ in the Brillouin zone. From bottom to top: $q=0.25 \pi / d, 0.50 \pi / d, 0.75 \pi / d$, and $1.0 \pi / d$.

sponding to the $n=1$ and 2 peaks in Fig. 1 (the $n=0$ peak of Fig. 1 being absent).

This analysis shows that in this method a measurement of the density $|\Psi(\Delta z, t)|^{2}$ in a portion $\Delta z$ of the BEC could reveal excitations in the higher bands, but should have no access to those in the lowest band. This is in accord with the aforementioned theoretical result [11] showing that excitations in the $n=0$ band correspond to pure phase modulations. In the kicking process a phase modulation of the condensate is excited, and only a measurement of a phaserelated quantity, such as the BEC average velocity, may give the desired information in the lowest-energy band.

In an actual experiment the kicking method may be realized by suddenly accelerating the optical lattice on a microsecond time-scale. A measurement of the BEC momentum distribution $n(p, t)=\left|\int d z \Psi(z, t) \exp (i p z)\right|^{2}$, after variable intervals of time $t$ by a time-of-flight technique, would give spatial variations of the BEC phase through the average velocity $\langle v\rangle=\int d z(\hbar p / M) n(p, t)$ [3]. An example of the measurement of the momentum distribution will be illustrated in Sec. IV below.

\section{B. Probing the density spectrum}

We turn to the second simulational method, that we refer to as shaking. In this method we modulate the BEC in space and time by parametrically driving the lattice potential. To this end we set $U_{p}(z, t)=\alpha U_{l}(z) \cos (q z-\Omega t)$ for $t<t_{d}$ and $U_{p}=0$ otherwise. We choose a small pump amplitude ( $\alpha$ $=0.15$ ), match the pump frequency $\Omega$ near resonance, and tune the drive time $t_{d}$ over several excitation periods, the time unit being $T_{R} \equiv h / E_{R} \simeq 0.32 \mathrm{~ms}$. The density is then recorded at times $t \gg t_{d}$.

Figure 2 displays the bulk density spectrum $|\Psi(z, \omega)|^{2}$ for the same $q$ values as in Fig. 1. The spectrum contains a peak centered at energy $\mu$ for all values of $q$ and a strong peak corresponding to the nearly resonant drive, at a frequency 


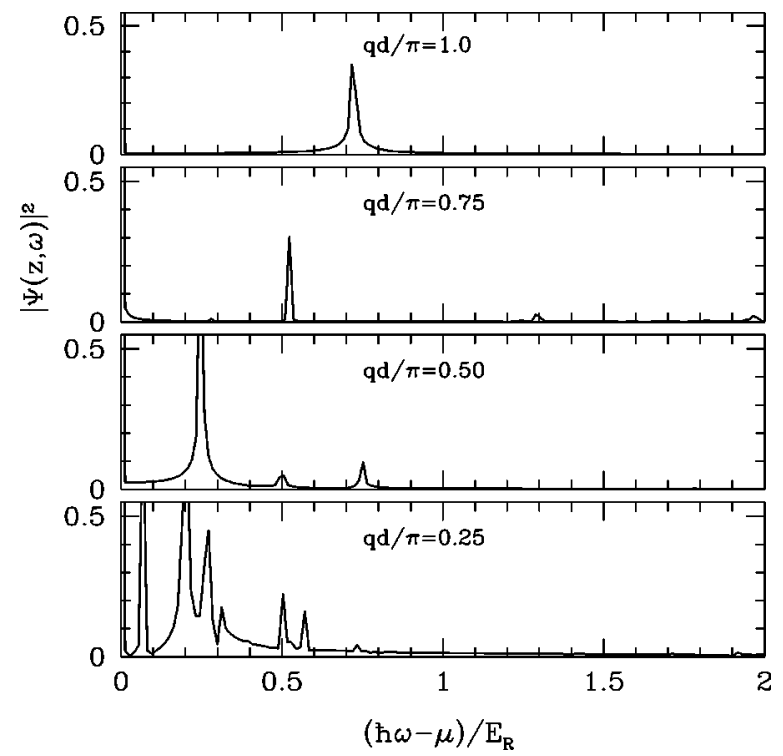

FIG. 2. Shaking method: bulk density $|\Psi(z, \omega)|^{2}$ as a function of $(\hbar \omega-\mu) / E_{R}$ for the same $q$ values as in Fig. 1 .

changing with $q$ in accord with the $n=0$ peaks in Fig. 1 . The other structures in Fig. 2 can be identified as harmonics of the fundamental excitation energy, and combinations of them.

Thus a near-resonance measurement of the density $|\Psi(z, t)|^{2}$ in the shaking method may reveal the excitations in all bands, and in particular those in the $n=0$ band. This is possible since both the density and phase are excited during the parametric drive. Shaking at $q=0$ coherently drives the bound states in each well, and creates pure density fluctuations. Phase modulations are also triggered at $q \neq 0$, due to the spatial periodicity of the BEC: this is at variance with the shape-deformation modes in harmonic traps [18]. The energies of these phase and density excitations are located at the poles of the response function to the external drive, and therefore show up as peaks in $|\Psi(z, \omega)|^{2}$.

In an actual experiment the time driving of the lattice amplitude by an appropriate modulation of the laser intensity is feasible in the proposed range of frequencies. The spatial modulation is harder to realize, at least over the whole Brillouin zone. As to detection after driving, the density of a portion $\Delta z$ of the BEC as large as ten lattice spacings $(\simeq 5 \mu \mathrm{m})$ can be probed by a technique of absorption imaging after ballistic expansion [17].

\section{Static deformation}

Before quantitatively comparing the results obtained by the above dynamical methods, we pause to present the band structure that we obtained by a static deformation method. In this method we imprint a phase modulation on the BEC ground state so as to generate a state having some overlap with a Bloch state of quasimomentum $q$. We then propagate this modulated BEC in imaginary time, and monitor the average energy $\langle E\rangle$ as the BEC returns to its ground state. Figure 3 shows $\langle E\rangle$ vs $i t \hbar / E_{R}$ for the same $q$ values as in Fig. 1. Two plateaus are met during this evolution: the first

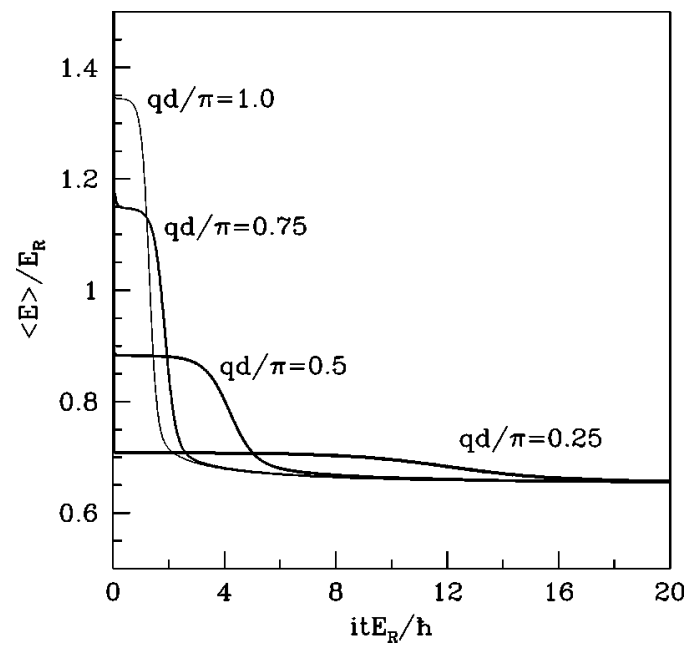

FIG. 3. Static deformation method: average energy $\langle E\rangle$ vs imaginary time $i t \hbar / E_{R}$ for a modulated BEC with the same $q$ values as in Figs. 1 and 2. Higher plateaus correpond to higher values of $q$ in the lowest band.

lies at an energy $E_{0}(q)$, and the second at an energy $\mu$. The time scales for the appearance of the plateaus correspond to interband and intraband relaxations, respectively. Similar results are obtained for the higher bands $E_{n}(q)$ from phase modulations having a wave vector $q+n^{\prime} \pi / d$ outside the first Brillouin zone (with $n^{\prime}=n$ for even $n$ and $n^{\prime}=n+1$ for odd $n)$.

The two-plateau structure in Fig. 3 results from a superposition of different Bloch states induced by the finite size of the simulation sample. An experimental equivalent would be a measurement of the release energy after imparting a velocity to the BEC as in the kicking method.

\section{Comparing the different methods}

We finally collect and compare the results analyzed in Secs. III A-III C. Figure 4 reports the energy bands $E_{n}(q)$ that we have obtained by the three methods presented above, for $n=0,1$, and 2 . The three methods yield the same excitation energies within the simulation accuracy, which is reflected by the size of the symbols (squares for the kicking method, circles for the shaking method, and triangles for the static method) in the main body of the figure. A gap has opened at $q=\pi / d$ between the $n=0$ and 1 bands, while there is no gap at $q=0$ between the $n=1$ and 2 bands: this is a well-known result for $1 \mathrm{D}$ lattice potentials having a doubled period [20]. We also remark that the results shown in Fig. 4 for the $n=1$ and 2 bands do not differ significantly from those that we obtain for a noninteracting BEC: indeed, with the present system parameters we have $U_{l}^{0} \simeq 2.12 \mu$, implying that the mean-field interactions are weak on the energy scale of the lattice.

The inset in Fig. 4 shows an enlarged view of the excitation energies in the lowest band up to $q d / \pi=0.5$, where the band starts to bend over. On this scale the estimated error bars of the simulation become visible, and well within them the results for the present interacting BEC agree with those for the noninteracting one, except perhaps at $q d / \pi=0.1$. The 


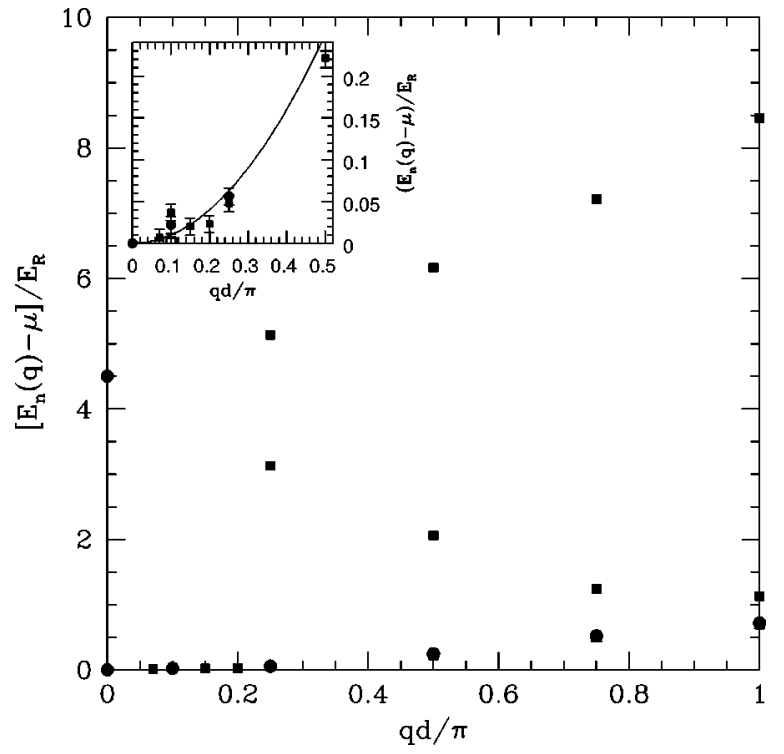

FIG. 4. The first three energy bands in the Brillouin zone 0 $\leqslant q \leqslant \pi / d$ as obtained by the methods reported in Figs. 1-3 (squares: kicking method; circles: shaking method; triangles: static method). The inset zooms in on the lowest band. The size of the error bars reflects a two-digit accuracy in energy differences. Solid line: quadratic dispersion with the bare atom mass.

calculated band energies are compatible with the freeparticle dispersion relation $\hbar^{2} q^{2} / 2 M$, as shown by the solid line in the inset. A high resolution, and a fine tuning of the strength of the self-interactions relative to the height of the lattice confinement, would clearly be needed to experimentally reveal the phononlike dispersion relation at long wavelengths which is predicted by gapless theories $[8,11]$. From the Bogolubov dispersion relation this linear behavior may be expected to become visible below $q \approx \sqrt{2} / \xi$.

\section{MEASURING THE MOMENTUM DISTRIBUTION}

We now discuss the momentum distribution $n(p, t)$ for a BEC in an optical lattice, as introduced in Sec. III A. We simulate a feasible experimental method for its measurement by driving a BEC with a force $F$ for a variable length of time $t_{d}$, as described in Ref. [3] for an experiment on ultracold atoms. The force is taken as a positive constant in the range $0 \leqslant t_{d} \leqslant T_{B} / 2$, and as a negative constant in the range $T_{B} / 2$ $<t_{d} \leqslant T_{B}$, with $T_{B}=h /|F| d$ being the period of Bloch oscillations. We set $U_{p}(z, t)=-F z$ for $t<t_{d}$ and $U_{p}=0$ otherwise, and choose the value of $F$ to correspond to a particle acceleration of $85 \mathrm{~cm} / \mathrm{s}^{2}$ as in the experiments of Ben Dahan et al. [3]. The Brillouin zone is explored in this method according to $q d / \pi=2 \operatorname{sgn}(F) t_{d} / T_{B}$.

Figure 5 shows $n(p, t)$ at times $0 \leqslant t_{d} \leqslant T_{B}$ covering one full Bloch oscillation. The peak seen at $t_{d}=0$ drifts to the right up to the first zone boundary at $t_{d}=T_{B} / 2$, while a second peak emerges in the second zone and moves to the left entering the first zone at later times. The sharpness of the peaks shows that the BEC is behaving as if it were a quasiparticle reflected back and forth at the Bragg planes. The inset in Fig. 5 shows that the BEC average velocity $\langle v\rangle$, as

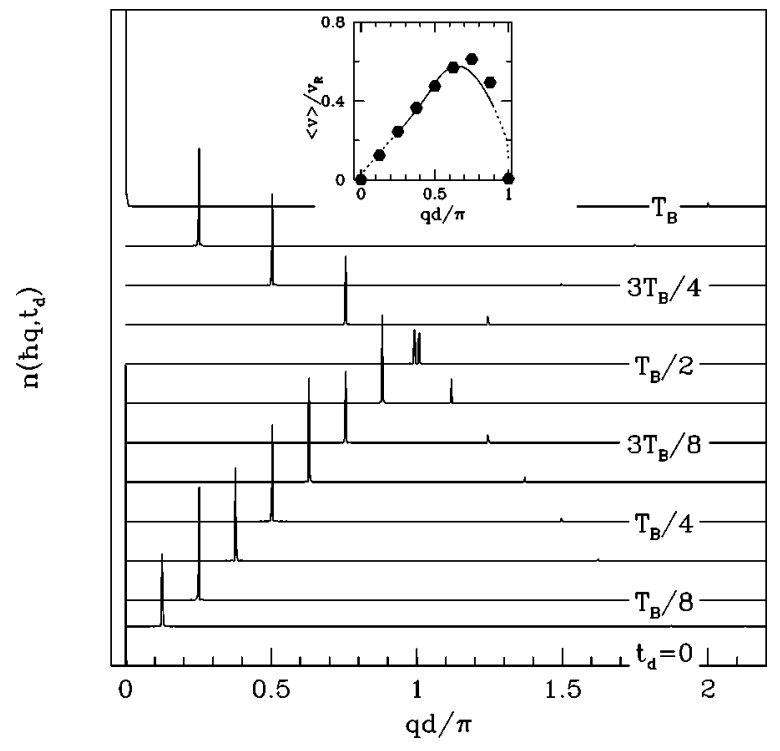

FIG. 5. Force-driving method: momentum distribution (in arbitrary units) at various times $t_{d}$, equally spaced by $T_{B} / 16$ up to $T_{B} / 2$ and then by $T_{B} / 8$ up to $T_{B}$. Inset: points give the BEC average velocity $\langle v\rangle$ (in units of $v_{R}=\hbar \pi / M d$ ) as determined from the momentum distribution after backfolding of the second Brillouin zone, while the line shows the semiclassical average velocity.

obtained from the momentum distribution after backfolding of the second zone, is consistent with the semiclassical average velocity as calculated from $d E_{0}(q) / d q$ using the results in Fig. 4.

\section{CONCLUDING REMARKS}

In conclusion, we have simulated three different experimental methods to probe the energy bands of a condensate in an optical lattice, and tested their meaning in terms of the density and phase deformations associated with excited band states. The velocity spectrum in the kicking method and the density spectrum in the shaking method are the correct obervables, from which the whole band structure can be explored and the nature of the BEC excitations inferred. The measurement of the momentum distribution within the forcedriving method will demonstrate the characteristic coherent dynamical behavior of the condensate.

We conclude by noting that a study of the observability of the expected linear dispersion at small $q$ values would require different system parameters than the present ones, and in particular stronger mean-field interactions and larger lattice constants. To this extent, the details of the overall confinement would become important, and should be appropriately tailored in the simulation.

\section{ACKNOWLEDGMENTS}

One of us (M.L.C.) thanks Dr. J. H. Müller for enlightening discussions on experimental issues. Part of this work was done using the computational facilities of Cineca. We acknowledge support from MURST through PRIN2000. 
[1] P.S. Jessen and I.H. Deutsch, Adv. At., Mol., Opt. Phys. 37, 95 (1996).

[2] Q. Niu, X.-G. Zhao, G.A. Georgakis, and M.G. Raizen, Phys. Rev. Lett. 76, 4504 (1996); S.R. Wilkinson, C.F. Bharoucha, K.W. Madison, Q. Niu, and M.G. Raizen, ibid. 76, 4512 (1996).

[3] M. Ben Dahan, E. Peik, J. Reichel, Y. Castin, and C. Salomon, Phys. Rev. Lett. 76, 4508 (1996); E. Peik, M. Ben Dahan, I. Bouchoule, Y. Castin, and C. Salomon, Phys. Rev. A 55, 2989 (1997).

[4] C.F. haroucha, K.W. Madison, P.R. Morrow, S.R. Wilkinson, B. Sundaram, and M.G. Raizen, Phys. Rev. A 55, R857 (1997).

[5] B.P. Anderson and M.A. Kasevich, Science 281, 1686 (1998).

[6] V. Vuletic, A.J. Kerman, C. Chin, and S. Chu, Phys. Rev. Lett. 82, 1406 (1999).

[7] G.K. Brennen, C.M. Caves, P.S. Jessen and I.H. Deutsch, Phys. Rev. Lett. 82, 1060 (1999).

[8] K. Berg-Sørensen and K. Mølmer, Phys. Rev. A 58, 1480 (1998).

[9] D. Jaksch, C. Bruder, J.I. Cirac, C.W. Gardiner, and P. Zoller,
Phys. Rev. Lett. 81, 3108 (1998).

[10] K.-P. Marzlin and W. Zhang, Phys. Rev. A 59, 2982 (1999).

[11] M.L. Chiofalo, M. Polini, and M.P. Tosi, Eur. Phys. J. D 11, 371 (2000).

[12] D.-I. Choi and Q. Niu, Phys. Rev. Lett. 82, 2022 (1999).

[13] M.L. Chiofalo, S. Succi, and M.P. Tosi, Phys. Lett. A 260, 86 (1999).

[14] M.L. Chiofalo and M.P. Tosi, Phys. Lett. A 268, 406 (2000).

[15] M.M. Cerimele, M.L. Chiofalo, F. Pistella, S. Succi, and M.P. Tosi, Phys. Rev. E 62, 1382 (2000).

[16] J.C. Bronski, L. Carr, B. Deconinck, and J.N. Kutz, e-print cond-mat/0010099.

[17] D.S. Jin, J.R. Ensher, M.R. Matthews, C.E. Wieman, and E.A. Cornell, Phys. Rev. Lett. 77, 420 (1996); D.M. Stamper-Kurn, H.-J. Miesner, S. Inouye, M.R. Andrews, and W. Ketterle, ibid. 81, 500 (1998).

[18] P.A. Ruprecht, M. Edwards, K. Burnett, and C.W. Clark, Phys. Rev. A 54, 4178 (1996).

[19] M.L. Chiofalo, S. Succi, and M.P. Tosi, Phys. Rev. E 62, 7438 (2000).

[20] J.C. Slater, Phys. Rev. 87, 807 (1952). 